\title{
Dislocation Engineered Silicon for Light Emission
}

\author{
K. P. Homewood ${ }^{1}$, M. A. Lourenço ${ }^{1}$, M. Milosavljevic1*, G. Shao ${ }^{2}$ and R. M. \\ Gwilliam ${ }^{1}$, \\ ${ }^{1}$ Advanced Technology Institute, School of Electronics and Physical Sciences, \\ University of Surrey, Guildford, Surrey, GU2 7XH, UK \\ ${ }^{2}$ BCAST, Brunel University, Uxbridge UB8 3PH, UK \\ *On leave from: VINCA Institute of Nuclear Sciences, Belgrade, Serbia and \\ Montenegro
}

\begin{abstract}
Although silicon is the preferred material for mainstream microelectronic applications, light emission from silicon is inefficient due to its indirect band gap. Despite this intrinsic problem, many approaches have been tried to obtain light emission in silicon-based devices. Porous silicon [1], silicon/silicon dioxide superlattices [2], silicon nanoprecipitates in silicon dioxide [3], erbium in silicon [4], silicon/germanium [5], and iron disilicide [6] are potential routes. However, a common problem found in these devices is the strong thermal quenching of the luminescence leading to very poor performance at room temperature.
\end{abstract}

$\mathrm{Ng}$ et al. [7] proposed a new method for device fabrication, the dislocation engineered approach, in order to solve the general thermal quenching problem of luminescence frequently found in semiconductors. In this approach, the thermal quenching giving poor room temperature luminescence can be minimised by the controlled introduction of dislocation loops. This introduces a strain field outside the loop that increases the silicon band gap by up to $0.75 \mathrm{eV}$ and enables spatial confinement of the injected carriers, so diffusion of injected carriers to point defects and the surface, where efficient non-radiative recombination occurs, is suppressed or eliminated.

The standard dislocation engineered light emitting diodes (DELEDs) operate with an emission wavelength at the short wavelength end of the extended optical communication band, $1.2 \mu \mathrm{m}$. There is considerable interest in other wavelengths particularly from 1.3 to $1.6 \mu \mathrm{m}$ for optical fibre communications. One way to obtain these wavelengths is to use other optically active materials or centres in silicon. However this approach has always failed in silicon due to the thermal quenching problem mentioned earlier. However in a DELED the active region of the device has been decoupled from the regions containing non-radiative centres and so optically active materials or centres introduced in to the active region between the depletion region edge and the loops should avoid silicon related non-radiative recombination. The emission spectrum observed should then just be determined by the relative rates of the optical centre emission and the radiative silicon transition.

Previously, we reported a silicon/iron disilicide LED [6]] but strong thermal quenching made this an impractical device for high temperature operation. Comparison of an iron disilicide LED and an identical LED where dislocation loops have been introduced above the iron disilicide crystallites as a function of operating temperature gives around a five decade improvement in the thermal quenching behaviour! The residual quenching is attributed to non-radiative recombination at the 
iron disilicide grain boundaries in this particular device. The spectrum of this device shows emission at both $1.2 \mu \mathrm{m}$ (the silicon) and $1.5 \mu \mathrm{m}$ (the iron disilicide). The form of the spectrum and its temperature dependence can be fully modelled assuming interaction between the silicon and silicide only.

A centre of particular interest is the rare earth erbium, which has an internal transition at $1.55 \mu \mathrm{m}$. This has been extensively studied in silicon and again strong thermal quenching completely prevents the observation of luminescence at room temperatures

We have fabricated a DELED, with erbium incorporated in the active region. In this case not only has the thermal quenching been eliminated, but the erbium emission is actually highest at room temperature. The EL spectra can again be fully explained by simple competition between the silicon and erbium devices.

We outlined here a new approach dislocation engineering to make efficient light emitting diodes in silicon using otherwise conventional silicon processing. Progress on tuning these devices from 1.5 to $1.6 \mu \mathrm{m}$ by incorporation of additional optically active centres was demonstrated. This technology is likely to open up many new applications for silicon as a new optically emitting material.

\section{References}

[1] K.D. Hirschman, L. Tysbekov, S.P. Duttagupta, P.M. Fauchet, Nature 384 (1996) 338.

[2] Z.H. Lu, D.J. Lockwood, J.M. Baribeau, Nature 378 (1995) 258.

[3] T. Komoda, J. Kelly, F. Cristiano, A. Nejim, P.L.F. Hemment, K.P. Homewood, R. Gwilliam, J.E. Mynard, B.J. Sealy, Nucl. Inst.\& Meth. B 96 (1995) 387.

[4] B. Zheng, J. Michel, F.Y.G. Ren, L.C. Kimerling, D.C. Jacobson, J.M. Poate, Appl. Phys. Lett. 64 (1994) 2842.

[5] L. Vescan, T. Stoica, Journal of Luminescence 80 (1999) 485.

[6] D. Leong, M. Harry, K.J. Reeson, K.P. Homewood, Nature 387 (1997) 686.

[7] W.L. Ng, M.A. Lourenço, R.M. Gwilliam, S. Ledain, G. Shao, K.P. Homewood, Nature 410 (2001) 192. 